\title{
Chronometric Constructive Cognitive Learning Evaluation Model: Measuring the Consolidation of the Human Cognition Schema in Psychology Students' Memory
}

\author{
Guadalupe Elizabeth Morales-Martinez \\ IISUE, National Autonomous University of Mexico, Mexico City, Mexico \\ https://orcid.org/0000-0002-4662-229X \\ Maria Isolde Hedlefs-Aguilar \\ FIME, Nuevo Leon Autonomous University, Monterrey, Nuevo Leon, Mexico \\ https://orcid.org/0000-0003-1138-9835
}

Janneth Trejo-Quintana

IISUE, National Autonomous University of Mexico, Mexico City, Mexico https://orcid.org/0000-0002-7701-6938

Yanko Norberto Mezquita-Hoyos

Psychology department, Autonomous University of Yucatan, Yucatan, Mexico https://orcid.org/0000-0001-6305-7440

Miriam Sanchez-Monroy

Tecnologico Nacional de Mexico-Instituto Tecnologico de Merida, Yucatan, Mexico https:// orcid.org/0000-0001-5263-1216

\begin{abstract}
This study illustrates the application of the Chronometric Constructive Cognitive Learning Evaluation Model to assess learning about human cognition knowledge schema in 48 second-year psychology students $(79 \%$ females, $21 \%$ males). In the first phase, the participants carried out a conceptual definition task based on the Natural Semantic Networks technique. They defined ten target concepts related to the course by using verbs, substantives, adjectives, and pronouns (definers). Participants then rated the grade of relatedness between definers and targets concepts. Subsequently, the present authors carried out a computational simulation with data from the first study. In addition, students participated in a semantic priming experiment. They participated in a lexical decision task. Participants read pairs of words; these pairs were sometimes related by cognition scheme or common association, and sometimes were unrelated. The three tasks were applied at the start of the course and the end. The computational simulation analysis and ANOVA indicated that the initial pattern for conceptual
\end{abstract}


activation had changed at the end of the course. Additionally, the initial chronometric behavior of the human cognition schema of the participants also changed at the end of the course. This evidence supports the idea that cognitive evaluation tools can help assess the schematic behavior patterns induced by academic learning.

Keywords: academic learning; knowledge scheme; cognitive evaluation; semantic priming; psychology students

\section{Introduction}

Measuring learning is a core part of the academic life of students and teachers. Learning assessments can be a valuable means of developing, modifying, and improving the learning and teaching process. In this regard, Harlen (2007) emphasizes the importance of determining exactly what should be evaluated because such decision-making influences the creation of learning opportunities, the selection of the content to be taught, and the design of teaching aids. This intrinsic link between the assessment of learning and teaching allows students' skills and knowledge to be certified, providing feedback to teachers on each student's learning progress, and providing information on the effectiveness and quality of the educational system (Lambert \& Lines, 2000).

The effectiveness and impact of evaluation on teaching and learning depend on its validity, reliability, usefulness, and feasibility (Harlen, 2007). The design of evaluation instruments that are valid and reliable requires a clear definition of the phenomenon and a precise specification of which indicators related to the phenomenon should be measured. These two aspects are difficult to establish in academic learning since most of the mental objects of study in psychology (e.g., intelligence, emotions, consciousness), are intangible, and only indirect measures exist to observe them. The difficulties in defining learning and its cognitive indicators impacted its study in cognitive psychology. In this regard, Pozo (2006) mentions that the issue of learning measurement has rarely been explored from this perspective.

From the cognitive psychology perspective, the cognitive definition of learning involves at least two relevant aspects. The first is the processing which relates to how the individual receives the inputs, processes the information, and then generates an output. The output is the second aspect, whereby the result of the learning process becomes visible to the observer (e.g., through a motor response) or leads to an unobservable expression (e.g., cognitive change). In line with the second expression, Moreno (2010) defines learning as a permanent change in mental structures due to a person's interaction with the environment. Thus, academic learning can involve qualitative and quantitative changes in cognitive knowledge structures due to the experiences a student is exposed to through interaction with their academic environment. According to Bower (1975), learning declarative knowledge involves incorporating new information nodes or new relationships between them, producing changes in knowledge structures. Lopez et al. (2014) point out that learning also implies the modification of the relationships between the scheme's concepts and the changes in the weight of these relationships. 
According to this cognitive view of learning, Morales-Martinez et al. (2021) mentioned that academic learning evaluation should involve measuring changes in the organization of information, the mental structure and in the schematic behavior (in terms of time and dynamic) due to the exposure to learning experiences. The measurement of these learning aspects becomes even more relevant in the current context; as Arieli-Attali (2013) stated, the 21st-century society has an economy which is based on information. Hence, evaluation of the complex properties of knowledge is central to academic training for students. In this regard, cognitive psychology offers a wide range of techniques for measuring changes in different cognitive structures. In the human memory study, some techniques can measure changes in knowledge structures produced by knowledge acquisition in different contexts, including the classroom. One instance of this is the Chronometric Constructive Cognitive Learning Evaluation Model or C3-LEM (Lopez et al., 2014; Morales-Martinez \& Lopez-Ramirez, 2016; Morales-Martinez, 2020; Morales-Martinez et al., 2017; Morales-Martinez et al., 2015).

This model applies human information processing principles (serial and parallel) to explore and explain how a student's mind forms knowledge structures when studying a topic. In general, the C3-LEM proposes the combined use of chronometric and mental representation techniques to evaluate academic learning, taking into account the processes of selection, storage, and retrieval of information learned by students. The model comprises of two evaluation phases (Figure 1). The first refers to constructive evaluation of knowledge that implies the application of mental representation techniques and computational simulation to identify the evaluated knowledge scheme's organizational, structural, and dynamic properties. The second phase, the chronometric evaluation, involves the application of mental chronometry techniques that measure the degree of knowledge consolidation in a student's memory. The following sections present details on each evaluation phase.

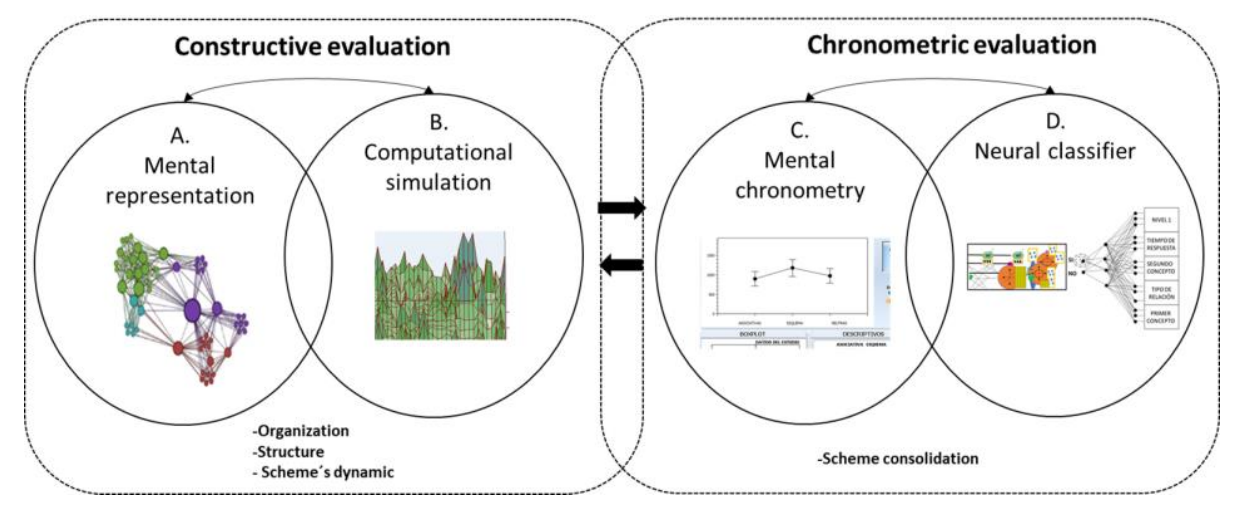

Figure 1: Phases and components of the C3-LEM

(Source: Morales-Martinez et al., 2020)

\section{Constructive Cognitive Assessment of Knowledge}

Constructive cognitive assessment refers measurement of the organization, structure, and dynamics of the learned knowledge scheme. The C3-LEM proposes techniques for evaluating the mental representation of knowledge, such as the 
Natural Semantic Networks (NSN), to explore how conceptual nodes are organized and structured in a student's memory. While studying the dynamics of mental structures of knowledge, this model suggests the inclusion of neural networks.

Figueroa et al. (1976) created the NSN to explore the meaning people give to an object (e.g., a career, a class). This technique contemplates a definitional task. Participants define a concept (target) with definers such as noun verbs, adjectives, or pronouns. The participants complete this process of specifying definers within a time window imposed by the researcher. Participants then weigh their definers in terms of the degree of relationship they perceive them to have with the target. Morales-Martinez et al. (2021) point out that some of the most relevant NSN technique indicators include the frequency of the occurrence of the definer, the semantic richness or J value, the $\mathrm{M}$ value or weight of semantic relevance, and the Inter-Response Time (IRT). IRTs refers to the time that passes in the recovery and writing of a definer. The authors make a special mention of this last indicator in the following section since this work illustrates the usefulness of chronometric indicators of knowledge. Previous work describes the other values (MoralesMartinez, Angeles-Castellanos, et al., 2020; Morales-Martinez et al., 2021). Additionally, a brief description of some NSN values is given in the data analysis section of this article.

Lopez-Ramirez et al. (2014) proposed using the NSN in the educational field to explore academic learning. They also suggested that the data obtained through this technique could help computer simulations of schemata behavior (LopezRamirez et al., 2015). This proposal gave way to the C3-LEM, which contemplates the application of NSN before and after students have taken a course, to extract data that allow computer simulations to emulate the knowledge scheme's behavior at the beginning and the end of the academic year. In addition, NSN provides information about the meaning that students attach to the knowledge learned (Morales-Martinez et al., 2015; Morales-Martinez et al., 2018; MoralesMartinez et al., 2021; Morales-Martinez \& Lopez-Ramirez, 2016; MoralesMartinez \& Santos-Alcantara, 2015).

Computational simulations can show how the schematic activity changes from the beginning to the end of the course, given academic learning. For example, Lopez et al. (2015) reported the changes in the scheme activation dynamic through different domains of knowledge such as customer service, information systems, and music. Moreover, computer simulations can also provide information about connections between concepts that are not easy to observe without these tools. For example, Gonzalez et al. (2013) explored the dynamics and emergence of high school students' moral knowledge schema. They observed that computational simulations could identify the information implicitly activated in a student's mind, activating concepts that were not necessarily the most relevant in the NSN. To illustrate this result, the authors reported that one parent's activation coactivated the concept police officer. The co-activation of this concept seems to have no semantic relationship with the first activated concept; however, when the 
researchers considered the participants' social and cultural context, they noted a relationship of psychological meaning.

In general, the NSN and the computational simulations provide indicators that allow the changes in the mental representation that students form during learning for a topic to be analyzed. The combination of NSN with experimental cognitive studies empowers researchers to observe different cognitive aspects of learning. For example, the inclusion of experiments based on the semantic priming paradigm could provide information on a schema's chronometric properties. It could help form a more integrative view of students' learning processes.

\section{Chronometric Cognitive Assessment of Knowledge}

Chronometric cognitive assessment of learning involves measuring the temporal patterns of schematic activity. The C3-LEM provides several chronometric indicators, such as the IRT; this is the time it takes for a definer to appear within an NSN. The IRT can provide information on the cognitive nature of the concepts. For example, Morales-Martinez and Santos-Alcantara (2015) reported how the definers concepts with the greatest semantic weight tend to appear between 30 and 40 seconds, occupying between the third and fifth positions on the list. Morales-Martinez (2020) surmised that this result might suggest that the M value may be more related to the schema's organization properties than the ease with which students access the schema content.

On the other hand, cognitive evaluation includes learning indicators as the reaction times (RT); these are generally obtained from the application of semantic priming studies. After this, a neural network classifies the RT to discriminate whether the students had integrated the academic content learned during a course into their long-term memory structures at the end of the course. The main proposal of the semantic priming paradigm' is that the recognition patterns for the stimuli (semantic or eidetic) are affected by the context that precedes them. For example, when presenting a stimulus (word or image) which is preceded by another semantically related stimulus, people will recognize the second stimulus more quickly or accurately compared to when there is no semantic relationship between both stimuli. Generally, semantic priming is explored through lexical decision tasks (McNamara, 2005); these involve the presentation of pairs of words with different relationships. The participant's task is to read the first word (prime) and then read the last word (target) and decide if it is a word or non-word. Target word recognition times can indicate whether there is a semantic relationship between it and the target.

The recognition times, in the categorization of the word pairs with a schematic relationship, are of particular interest in C3-LEM studies because they provide information about the consolidation of the knowledge schema in memory. C3LEM proposes that semantic relationships between the word pairs related to the evaluated knowledge schema do not exist in the student's memory before the course, so the target recognition times in the schematic pairs will be significantly different at the beginning of the course compared to at the end. Also, these will 
differ from those obtained in the targets of word pairs with other types of semantic relationships (e.g., associative, categorical, or unrelated).

The semantic priming effect produced by a schematic relationship is called "schematic priming" (Lopez, 1996; Lopez \& Theios, 1992). In academic learning, schematic priming is present in word recognition tasks related to knowledge schema that the student is learning on a course. When a student stores the conceptual nodes learned in class in long-term memory, the recognition times for schema words have decreased by the end of the course (e.g., Gonzalez et al., 2013; Morales-Martinez, Lopez-Perez, et al., 2020). The opposite happens when students do not consolidate the information learned in their memory (MoralesMartinez, Angeles-Castellanos, et al., 2020).

On the other hand, some studies indicate that spatial strategies can affect the recognition times for schematic words. For example, Urdiales-Ibarra et al. (2018) explored the use of concept maps to reinforce the learning of a biology scheme among high school students. They observed that only the group that used the concept maps obtained a schematic priming effect in recognizing words associated with the course. In contrast, the group which did not use maps, seem not to have consolidated the information in their memory. From the present authors' point of view, greater experimental controls in spatial strategies are lacking in this study.

The exploration of new knowledge domains with C3-LEM studies can enhance the comprehension of the cognitive process through which students' progress in their conceptual understanding in a domain of knowledge. These studies can also provide information on the learning strategies that students use to expand their learning. Thus, this work aimed to contribute to the formation of this body of empirical evidence by exploring the cognitive properties of a knowledge scheme on human cognition in psychology students. In particular, the main objective of this study was to explore the cognitive changes on evaluated schema as a result of academic learning. When students learn information from a course, the time it takes them to access and retrieve this information from memory decreases from the beginning of the course to the end. Thus, the authors looked for changes in the temporal patterns relating to recovering information. Moreover, academic learning should influence schema activation, and so the initial conceptual activations at the beginning of the course should be different those at the end. Bearing this in mind, another objective of this study was to observe variations in the definers' initial activity at the end of the course. Finally, if students consolidated the course information in their long-term memory, then they should cognitively access this information more quickly at the end of the course compared with the beginning. Then, a final objective was to determine if the recognition time for schema words was faster at the end of the course than the beginning of the course. 


\section{Method}

\subsection{Study Design}

The authors applied the NSN technique to observe behavior related to a human cognition scheme and carried out a computational simulation based on Lopez and Theios (1992). Subsequently, to explore the temporal properties of the participants' knowledge schema on human cognition, the researchers designed an experimental study based on the semantic priming paradigm with a lexical decision task.

\subsection{Participants}

The participants were 48 second-year psychology students who took a course on human cognition. The participants' ages ranged from 19 to 34 years old ( $\mathrm{M}=20.3$, $\mathrm{SD}=2.5)$; of these, $38(79 \%)$ were females, and $10(21 \%)$ were males. All the students were volunteers and gave informed consent to participate.

\subsection{Instruments and Materials}

The authors obtained a list of definers with the Protocol for the Collection of Target Concepts and Central and Deferred Definers (Morales-Martinez, 2015). This protocol is a procedure that allows the teacher to select target and definer concepts that are central to the development of the course. The selected definers were organized into pairs of prime-target stimuli (e.g., cognition-conditional, cognitive-heuristic, perception-supervisor). The authors used these prime-target pairs to form the experimental conditions for the semantic priming study. Also, the researchers used EVCOG software to design, apply, and analyze data from mental representation and semantic priming studies. The EVCOG also allows computational simulations to be carried out on the NSN data (Morales-Martinez, Angeles-Castellanos et al., 2020).

\subsection{Procedure}

Students performed a concept definition task based on the NSN technique (Morales-Martinez et al., 2021). Participants had to define ten target concepts related to human cognition schema. They used nouns, verbs, and adjectives as definers to define each target concept. Participants then rated each definer on a 10-point scale based on the degree of relatedness to the target that the participants perceived the definer to have. While 1 represented a weak definer or a poor relationship to the target concept, 10 indicated a definer closely related to the target concept. Subsequently, the researchers applied a Boltzmann neural network with constraints satisfaction to the NSN study data. First, the researchers used the EVCOG software to obtain the association weights matrix between the definers. The software calculates the probability that two definers co-occur, through a modified algorithm from Rumelhart et al. (1986) by Lopez and Theios (1992) and Lopez (1996).

During the second phase, students performed a lexical decision task based on the semantic priming paradigm. First, students participated in a practice session to become familiar with the experimental activity. Later, the participants performed the experimental task. Each experimental condition consisted of pairs of words organized with an experimental sequence illustrated in Figure 2. First, a dot 
appeared in the center of the computer screen for 500 milliseconds; it centered each participant's attention. Subsequently, a prime appeared and remained on the screen for 250 milliseconds. Then, a blank space appeared for 50 milliseconds. Finally, the target appeared on the screen and remained until the participant had performed the experimental task. The task consisted of silently reading the prime and the target and then deciding whether the latter was well written. The study duration for each participant ranged from 7 to 8 minutes, depending on their characteristics.

SOA: $300 \mathrm{~ms}$

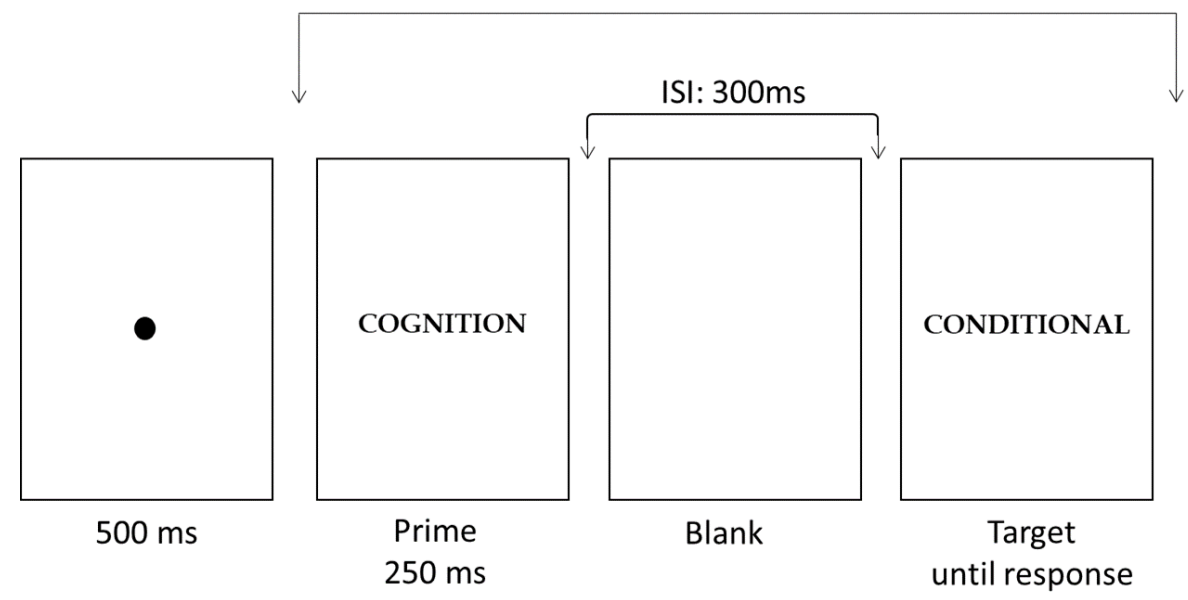

Figure 2: A sequence of an experimental trial in the semantic priming study Note: SOA = Stimulus Onset Asynchrony, ISI = Inter Stimulus Interval

\section{Analyzing the Data}

Three analyses were carried out on participants' data. First, the authors made a visual inspection of the distribution of IRTs obtained from the definers in all NSN. Then, the researchers explored the temporality pattern of the definers of the target with greater semantic richness, in line with Morales-Martinez et al. (2021). Semantic richness or $\mathrm{J}$ value refers to the number of definers that a person can generate to represent an object. Subsequently, a computational simulation was carried out on the concepts with the highest $M$ values. The $M$ value refers to the quantitative judgment that a participant makes about the semantic relevance that each definer has in representing the evaluated target. Finally, the authors applied a repeated-measures ANOVA on the data from the semantic priming study.

\section{Results}

\subsection{Qualitative analysis of the IRT of the NSN concepts}

The authors visually inspected the inter-response times or IRTs to observe the temporal pattern for definers before and after the course. Figure 3 shows that the distribution bias of the IRTs contracts from right to left. This finding implies a decrease in the recovery time for the definers after the course. Also, note that the $\mathrm{M}$-value for many concepts had increased after the course. 

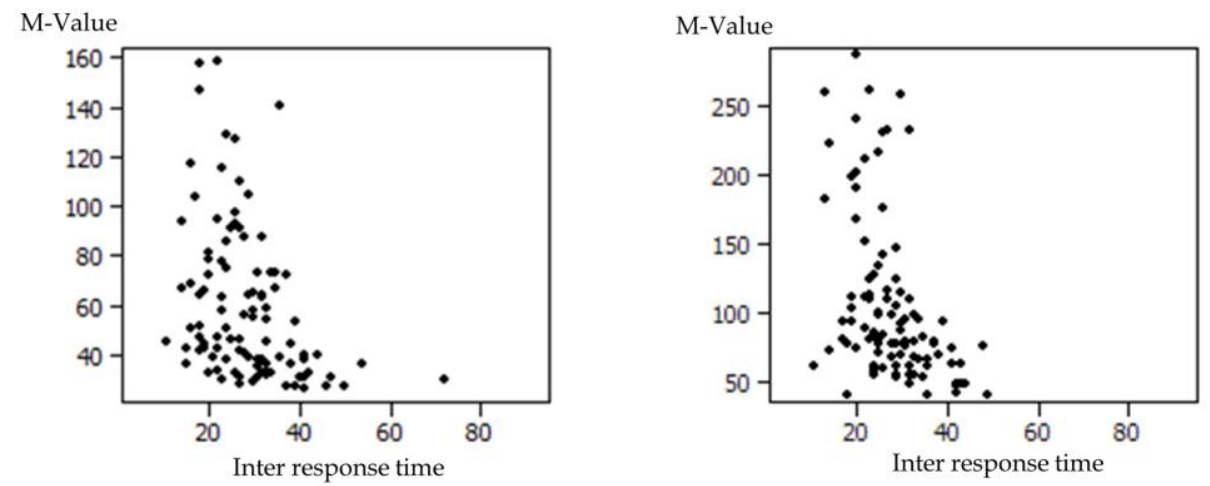

Figure 3: Inter-response times for the definers before and after the course

On the other hand, Figure 4 compared the IRT patterns obtained at the beginning and end of the course between concepts with the highest and lowest semantic weight reported in Morales-Martinez et al. (2021).
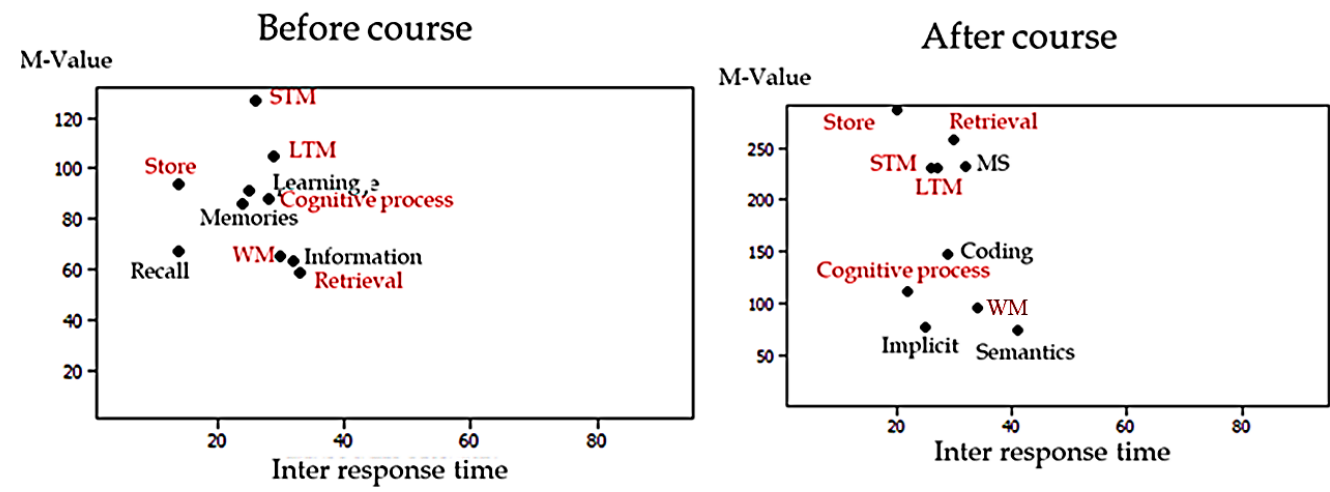

a) Memory
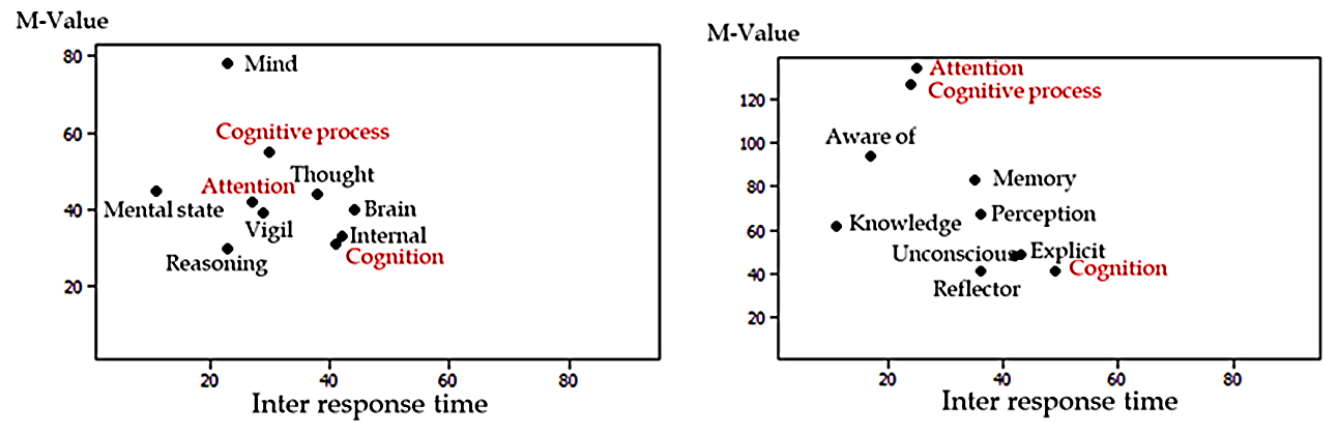

b) Consciousness

Figure 4: Comparison between definers' inter-response times for the target a) with greater and b) less semantic richness at the beginning and end of the course

Figure 4 reveals that the targets with the greatest and the least semantic richness (memory and consciousness) had changes in the content and relevance weighting for definers. For example, although the students conserved the $60 \%$ of initial definers to define memory, they showed a possible change in their understanding 
of the meaning of this target; at the beginning of course, they gave greater weight to definers related to structure, while at the end of course, students include, among the most relevant definers, those related to the process and structure of human memory. Additionally, there was a change in the IRT in terms of recovery for the definers. For example, at the end of the course, the students gave greater semantic relevance to the definers of storage and retrieval. In both cases, the retrieval time window changed at the end of the course. Also, the common definers of consciousness (attention, cognitive process, cognition) changed their semantic weight (J value) and their access time at the end of the course.

\subsection{Computational simulation}

A computational simulation was carried out using data from the previous study by Morales-Martinez et al. (2021). The objective was to observe the schematic behavior of the definer concepts with the highest semantic weight (Table 1).

Table 1. Definers and semantic weights were obtained in the study by MoralesMartinez et al. (2021)

\begin{tabular}{|c|c|c|}
\hline \multicolumn{3}{|c|}{ Before course } \\
\hline Target & Definer & $\mathbf{M}$ \\
\hline Cognitive psychology & Cognitive process & 159 \\
\hline Cognition & Cognitive process & 158 \\
\hline Attention & Cognitive process & 129 \\
\hline Memory & STM & 127 \\
\hline Reasoning & To think & 117 \\
\hline Decision making & Choice & 104 \\
\hline Perception & Sense & 97 \\
\hline Representation & Schemata & 95 \\
\hline Consciousness & Mind & 78 \\
\hline Problem solving & Reasoning & 64 \\
\hline \multicolumn{3}{|c|}{ After course } \\
\hline Target & Definer & $\mathbf{M}$ \\
\hline Cognitive psychology & Cognitive process & 230 \\
\hline Cognition & Cognitive process & 259 \\
\hline Attention & Filter & 211 \\
\hline Memory & Store & 286 \\
\hline Reasoning & Reasoning & 239 \\
\hline Decision making & Choice & 222 \\
\hline Perception & Sensation & 182 \\
\hline Representation & Schemata & 261 \\
\hline Consciousness & Attention & 134 \\
\hline Problem solving & objective & 114 \\
\hline
\end{tabular}


The definers with the highest semantic weight at the end of the course were activated with the restrictions satisfaction's neural net. Then, the authors observed the co-activation pattern. Tables 2 and 3 show the co-activations obtained to the definer clamped of cognitive process. The authors selected this definer because it has the highest appearance frequency and is among the definers with the highest $\mathrm{M}$ value, according to the NSN, at the beginning and the end of the course.

Table 2. Co-activated definitions for the stapling concept of cognitive processes before the course

\begin{tabular}{|c|c|c|c|}
\hline Definer & Activation & Definer & Activation \\
\hline Store & * & STM & - \\
\hline Analysis & - & Memory & * \\
\hline Learning & * & Mental & - \\
\hline Attention & - & Mind & * \\
\hline Selective attention & - & LTM & * \\
\hline Capacity & * & Models & - \\
\hline Brain & * & WM & * \\
\hline Science & - & Object & - \\
\hline Cognition & * & Observe & * \\
\hline Concentration & - & Options & * \\
\hline Concepts & * & Thought & - \\
\hline Behavior & - & Think & - \\
\hline Consciousness & - & Perception & - \\
\hline Choice & - & Processing & - \\
\hline Schemata & - & Cognitive process & * \\
\hline State of mind & - & Reasoning & - \\
\hline Stimuli & * & To reason & * \\
\hline Study & * & Reality & - \\
\hline Evaluation & - & Remember & - \\
\hline Focus & * & Memories & * \\
\hline Human & - & Recovery & - \\
\hline Imagen & * & Senses & * \\
\hline Information & * & Sense & - \\
\hline Internal & * & Symbols & - \\
\hline Interpretation & * & Solutions & * \\
\hline Logic & * & Vigil & * \\
\hline
\end{tabular}


Table 3: Co-activated definitions for the stapling concept of cognitive processes after the course

\begin{tabular}{|c|c|c|c|c|c|}
\hline Definer & Activation & Definer & Activation & Definer & Activation \\
\hline Store & * & Implicit & * & Syllogism & - \\
\hline Alternatives & - & Unconscious & * & Normative & - \\
\hline Analogical & - & Inductive & * & Threshold & - \\
\hline Hot & * & Information & * & & \\
\hline Attention & - & Interpret & * & & \\
\hline Divided & * & Logic & * & & \\
\hline Sustained & - & LTM & - & & \\
\hline Absence & * & Memory & * & & \\
\hline Capacity & * & Mind & - & & \\
\hline Science & * & Goal & * & & \\
\hline Coding & * & STM & - & & \\
\hline Cognition & * & Attenuation & - & & \\
\hline Cold & - & STM & * & & \\
\hline Concepts & - & WM & - & & \\
\hline Conclusions & * & Neisser & * & & \\
\hline Knowledge & - & Objective & * & & \\
\hline Realize & * & Choices & - & & \\
\hline Decision & - & Direct & - & & \\
\hline Choice & - & Perception & * & & \\
\hline Schemata & - & HIP & * & & \\
\hline Initial state & - & Premises & - & & \\
\hline Stimuli & * & Problem & * & & \\
\hline Strategies & - & Processing & - & & \\
\hline Evaluation & - & Process & * & & \\
\hline Experience & - & Psychology & * & & \\
\hline Explicit & - & Reasoning & * & & \\
\hline Filter & - & Deductive & - & & \\
\hline Focus & - & Retrieve & * & & \\
\hline Gestalt & * & Reflector & * & & \\
\hline Heuristics & * & Representation & * & & \\
\hline Human & - & Selective & * & & \\
\hline Illusion & * & Semantics & - & & \\
\hline Images & - & Sensation & * & & \\
\hline To imagine & * & Senses & - & & \\
\hline
\end{tabular}

Note that in the first and second phases, definers with implicit relationships and those closely related to the activated concept were co-activated. However, during the first phase, the number of definers related implicitly to the activated target was greater than during the second phase. In the second part of the evaluation, the computer simulation resulted in a balanced activation of definers with implicit and direct relationships.

\subsection{Semantic Priming Study}

The authors conducted a repeated-measures ANOVA of 2 (course time: beginning vs. end of the course) $\times 3$ (semantic relationship: associative vs. schematic vs. 
unrelated pairs of words) on the participants' data (Table 4), with $p \leq .01$ as the significance level.

Table 4: ANOVA of repeated measures between the factors of temporal relation and state of knowledge

\begin{tabular}{|c|c|c|c|c|c|c|c|}
\hline \multirow[t]{2}{*}{ Factor } & \multicolumn{2}{|r|}{ Effect } & \multicolumn{2}{|r|}{ Error } & \multirow[t]{2}{*}{$F$} & \multirow[t]{2}{*}{$p$} & \multirow[t]{2}{*}{$\eta_{p}^{2}$} \\
\hline & $d f$ & $M S$ & $d f$ & $M S$ & & & \\
\hline Course time (CT) & 1 & 1454649.388 & 47 & 23443.495 & $62.049^{*}$ & .001 & .56 \\
\hline Semantic relation $(\mathrm{ST})$ & 2 & 1759834.347 & 94 & 9784.957 & $179.851^{*}$ & .001 & .79 \\
\hline $\mathrm{CT} * \mathrm{ST}$ & 2 & 83875.5138 & 94 & 4362.258 & $19.227^{*}$ & .001 & .29 \\
\hline
\end{tabular}

Note: $\mathrm{N}=48$. ANOVA = analysis of variance; $\mathrm{df}=$ degree of freedom; $\mathrm{MS}=$ mean square; $\eta p 2$ = partial eta squared. ${ }^{*} \mathrm{p} \leq .001$

The most relevant factor for word recognition was the semantic relationship $\left(\eta_{p}{ }^{2}=.72\right)$. The difference in word recognition time between the beginning and the end of the course was more significant in the schematic condition (Figure 5).

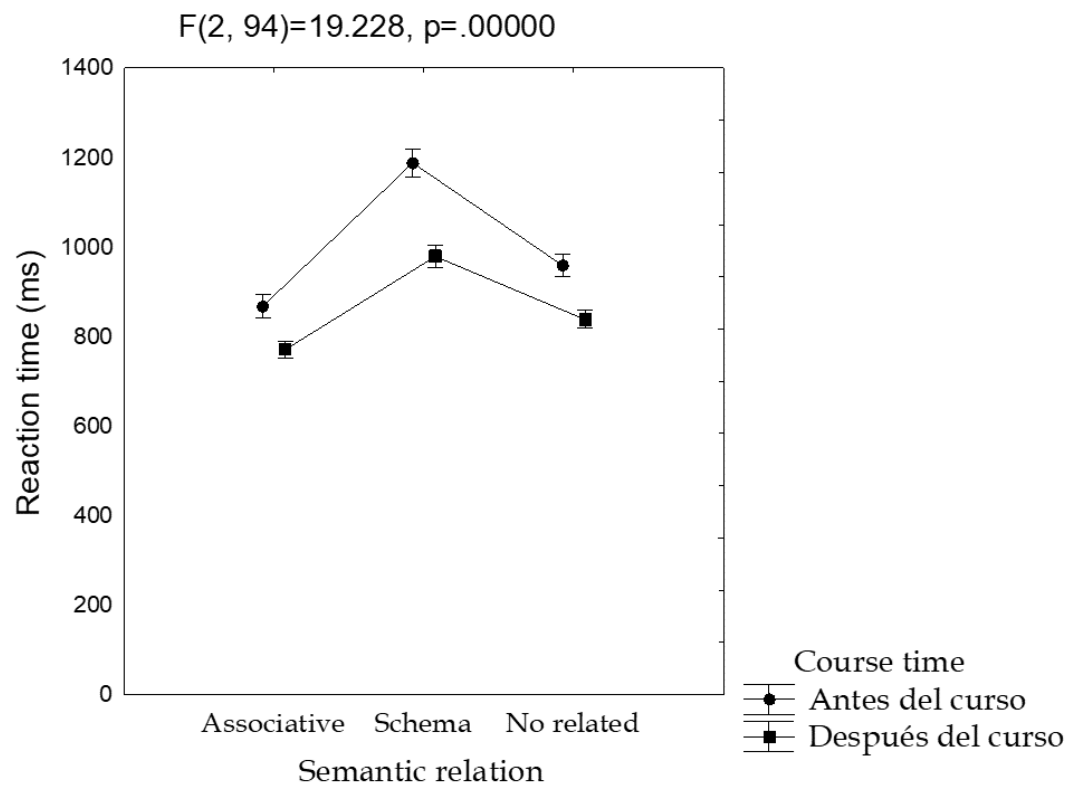

Figure 5: Interaction graph between the semantic relationship factor and the course time factor

\section{Discussion}

In cognitive psychology, student learning involves changes in the organization, structure, dynamics, and temporality of the knowledge schemas students learn on a course. In this work, the main objective was to evaluate, through the C3-LEM, the dynamics and temporality dimensions of the schema on human cognition that psychology students learned on a course.

In the NSN study, four different analyses were carried out; three were presented in the previous article by Morales-Martinez et al. (2021). This article presented the analysis of the appearance time patterns for the definers for each objective. In general, the results showed a shortening of the IRT window towards the end of the course (Figure 3). Other studies with similar data have not discussed the 
implications of these findings. However, the present authors suggest that this change in temporal pattern may indicate that the learning of a schema impacts the level of accessibility for the information stored in the student's memory.

Another interesting observation is that the IRTs for the definer concepts with the highest $\mathrm{M}$-value seem to require an access time of between 20 and 40 seconds, and they tend to appear between the 3rd and 5th position in the list of target concepts. So, although these types of definers are considered the most highly relevant, they are not the ones that are accessed the fastest. Morales-Martinez and SantosAlcantara (2015) obtained similar results; however, these authors did not hypothesize about this result. From the present authors' point of view, the definers with the highest $M$ value may take time to appear because they have a more significant cognitive load. That is, definers that have a greater weight in the NSN may take longer to appear because of the number of concepts with which they are associated or connected. Another possibility is that the $\mathrm{M}$ value indicates the degree of complexity of the definer.

On the other hand, the computational simulations indicated that the co-activation dynamics of knowledge schema evolve over the duration of the course. For example, the computer simulation activated a large number of definers with implicit relationships with the target at the beginning of the course. In contrast, the number of activated definers with closer theoretical relationships to the target had increased by the end of the course, although definers with implicit associations continued to appear throughout the network. The present authors hypothesize that this may agree with the results of the previous study (MoralesMartinez et al., 2021), in which the semantic network changed from one with a general approach to one where the student made use of more specific and specialized concepts.

Finally, the reaction time analyses provided information on the changes in the chronometric behavior for the scheme learned by the students. The ANOVA indicated that there was a main effect due to the type of semantic relationship and also given the application time factor. There was a significant decrease in the recognition RTs in the schematic words, suggesting that the students stored conceptual nodes related to the human cognition schema in their long-term memory. Similar findings have been reported in various studies (e.g., Gonzalez et al., 2013; Morales-Martinez, Lopez-Perez, et al., 2020); this suggests a change in the level of consolidation of the scheme learned by the students.

\section{Conclusion}

The evaluation of learning is a cornerstone for enhancing students' learning. It can offer very valuable information to improve learning and teaching processes. However, commonly, learning evaluation tools are designed to indicate academic performance as a product while the cognitive processes involved in the academic learning cannot be explored through these types of tools. Thus, this research work contributes with a new cognitive tool to measure students' cognitive gestion on the information they receive and learn on a course. Here, the authors explored the learning process among psychology students enrolled on a course on human 
cognition. In order to achieve the outcome, we used NSN to observe changes in the schema structure, computational simulation to explore the schema dynamic and a semantic priming experimental technique to determine the consolidation of information in the memory of students.

The data suggest that the learning derived from a course can be observed through changes in the dynamics and schematic temporality. In this regard, the NSN study's temporal analysis indicates a change in the level of accessibility for the information stored in memory as a product of academic learning. Computer simulations suggest that the number of activated conceptual nodes changes as the course progresses. Additionally, the schema dynamics changed in terms of the level of generality of the co-activated concepts. Apparently, at the end of the course, the schema tends to appeal to more specific concepts but preserves information on implicit relationships throughout the schema. In other words, what has been learned is not lost but is modulated by new inputs. On the other hand, the results indicated a change in the recognition time for the schematic words, suggesting a consolidation of the course information in the student's longterm memory.

In sum, this study's findings suggest that using the C3-LEM in learning assessment can help cover needs that Arieli-Attali (2013) regard as central for $21^{\text {st }}$ century students in terms of their academic training, namely assessment focused on information processing skills without neglecting the knowledge measurement itself.

\section{References}

Arieli-Attali, M. (2013, October 20-25). Formative assessment with cognition in mind: The cognitively based assessment of, for and as learning [Paper presentation]. 39th Annual Conference: Educational Assessment 2.0: Technology in Educational Assessment, Tel Aviv, Israel. https://www.iaea.info/conference-proceedings/

Bower, G. H. (1975). Cognitive psychology: An introduction. In W. K. Estes (Ed.), Handbook of learning and cognitive processes: Introduction to concepts and issues (pp. 25-80). Lawrence Erlbaum Associates.

Figueroa, J. G., Gonzalez, E. G., \& Solis, V. M. (1976). An approach to the problem of meaning: Semantic networks. Journal of Psycholinguistic Research, 5(2), 107-115. https://doi.org/10.1007/BF01067252

Gonzalez, C. J., Lopez, E. O., \& Morales, G. E. (2013). Evaluating moral schemata learning. International Journal of Advances in Psychology, 2(2), 130-136. http://www.ijJpsychol.org/paperInfo.aspx?ID=1981

Harlen, W. (2007). Assessment of learning. Sage Publications.

Lambert, D., \& Lines, D. (2000). Understanding assessment: Purposes, perceptions, practice (1st ed.). Routledge. https:// doi.org/10.4324/9780203133231

Lopez, E. O. (1996). Schematically Related Word Recognition (Publication No. 9613356) [Doctoral dissertation, University of Wisconsin-Madison]. ProQuest Dissertations \& Theses Global.

Lopez, E. O, \& Theios, J. (1992). Semantic analyzer of schemata organization (SASO). Behavior Research Methods, Instruments, \& Computers, 24(2), 277-285. https://doi.org/10.3758/BF03203508 
Lopez, E. O., Morales, G. E., Hedlefs, I., \& Gonzalez, C. J. (2014). New empirical directions to evaluate online learning. International Journal of Advances in Psychology, 3(2), 40-47. https://doi.org/10.14355\&ijap.2014.0302.03.

Lopez, R. E. O., Morales, M. G. E., Hedlefs, A. M. I., Gonzalez, T. C. J., \& Moreno, M. A. P. (2015). Nuevas direcciones empiricas en la investigacion e innovacion de tecnologia educativa para la evaluacion del aprendizaje en linea: Una aproximación conexionista [New empirical directions in educational technology research and innovation for the evaluation of online learning: A connectionist approach]. UANL Science / Ciencia UANL, 18(71), 52-64.

McNamara, T. P. (2005). Semantic priming. Perspectives from memory and word recognition. Psychology Press Ltd.

Morales-Martinez, G. E. (2015). Protocolo para la recoleccion de conceptos objetivo y definidores centrales y diferidos (PRECODECD): Un sistema de codificacion de conceptos extraidos de las redes semanticas naturales [Protocol for the collection of objective concepts and central and deferred definers (PRECODECD): A coding system for concepts extracted from natural semantic networks] [Unpublished manuscript]. Institute of Research on the University and Education, National Autonomous University of Mexico.

Morales-Martinez G. E. (2020). Sistema de evaluacion cognitiva constructiva cronometrica del aprendizaje en linea y presencial [Online and face-to-face learning's constructivechronometric cognitive assessment system] [Manuscript submitted for publication]. Institute of Research on the University and Education, National Autonomous University of Mexico.

Morales-Martinez, G. E., Angeles-Castellanos, A. M., Ibarra-Ramirez, V. H., \& ManceraRangel, M. I. (2020). Cognitive e-tools for diagnosing the state of medical knowledge in students enrolled for a second time in an anatomy course. International Journal of Learning, Teaching and Educational Research, 19(9), 341-362. https://doi.org/10.26803/ijlter.19.9.18

Morales-Martinez, G., \& Lopez-Ramirez, E. (2016). Cognitive responsive e-assessment of constructive e-learning. Journal of e-Learning and Knowledge Society (Je-LKS), 12(4), 39-49. https://doi.org/10.20368/1971-8829/1187

Morales-Martinez, G. E., Lopez-Perez, R. M., Garcia-Collantes, A., \& Lopez-Ramirez, E. O. (2020). Evaluacion constructiva cronometrica para evaluar el aprendizaje en linea y presencial [Chronometric constructive assessment to assess online and face-toface learning]. Technology, Science and Education / Tecnología, Ciencia y Educación, 15(1), 105-124. https://doi.org/10.51302/tce.2020.371

Morales-Martinez, G. E., Lopez-Ramirez, E. O., Castro-Campos, C., Villarreal-Trevino, M. G., \& Gonzales-Trujillo, C. J. (2017). Cognitive analysis of meaning and acquired mental representations as an alternative measurement method technique to innovate e-assessment. European Journal of Educational Research, 6(4), 455-464. https:// doi.org/10.12973/eu-jer.6.4.455

Morales-Martinez, G. E., Lopez-Ramirez, E. O., Garcia-Duran, J. P., \& Urdiales-Ibarra, M. E. (2018). Cognitive constructive - Chronometric techniques as a tool for the eassessment of learning. International Journal of Learning, Teaching and Educational Research, 17(2.), 159-176. https://doi.org/10.26803/ijlter.17.2.10

Morales-Martinez, G. E., Lopez-Ramirez, E. O., \& Lopez-Gonzalez, A. E. (2015). New approaches to e-cognitive assessment of e-learning. International Journal for eLearning Security (IjeLS), 5(2), 449-453. https://doi.org/10.20533/ijels.2046.4568.2015.0057

Morales-Martinez, G. E., Trejo-Quintana, J., Charles-Cavazos, D. J., Mezquita-Hoyos, Y. N., \& Sanchez-Monroy, M. (2021). Chronometric Constructive Cognitive Learning Evaluation Model: Measuring the Construction of the Human Cognition Schema of Psychology Students. International Journal of Learning, Teaching and Educational Research, 20(2), 1-21. https://doi.org/10.26803/ijlter.20.2.1 
Morales-Martinez, G. E., \& Santos-Alcantara M. G. (2015). Alternative empirical directions to evaluate schemata organization and meaning. Advances in Social Sciences Research Journal, 2(9), 51-58. https://doi.org/10.14738/assrj.29.1412.

Moreno, R. (2010). Educational psychology. John Wiley \& Sons, Inc.

Pozo, J. I. (2006). Teorias cognitivas del aprendizaje [Cognitive theories of learning] (9th ed.). Morata.

Rumelhart D., Smolensky P., McClelland, J., \& Hinton, G. (1986), Schemata and sequential thought processes in PDP models. In J. McClelland, D. Rumelhart \& the PDP Research Group (Eds.), Parallel distributed processing: Explorations in the microstructure of cognition: Psychological and biological models (pp. 7-57). MIT Press. https://doi.org/10.7551/mitpress/5236.003.0004

Urdiales-Ibarra, M. E., Lopez-Ramirez, E. O., Castro-Campos, C., Villarreal-Trevino, M. G., \& Carrillo-Colon, J. E. (2018). Biology schemata knowledge organization and meaning formation due to learning: A constructive-chronometric approach to concept mapping usability. Creative Education, 9(16), 2992-2706. https://doi.org/10.4236/ce.2018.916203 